\title{
Quantitative Electroencephalography Analyzed by Statistical Pattern Recognition as a Diagnostic and Prognostic Tool in Mild Cognitive Impairment: Results from a Nordic Multicenter Cohort Study
}

\author{
Malene Schjønning Nielsen ${ }^{a} \quad$ Anja Hviid Simonsen $^{b} \quad$ Volkert Siersma $^{c}$ \\ Knut Engedal ${ }^{d, e}$ Vesna Jelic $^{f}$ Birgitte Bo Andersen $^{b}$ Mala Naik ${ }^{e}$ \\ Steen Gregers Hasselbalch ${ }^{b}$ Peter Høgh ${ }^{a}$

\begin{abstract}
a Regional Dementia Research Centre, Department of Neurology, Zealand University Rigshospitalet, University of Copenhagen, Copenhagen, Denmark; ${ }^{C}$ The Research Unit for General Practice and Section of General Practice, Department of Public Health, University of Copenhagen, Copenhagen, Denmark; ${ }^{d}$ Norwegian Advisory Unit for Ageing and Health, Vestfold Health Trust, Toensberg, Norway; ${ }^{e}$ Department of Geriatric Medicine, Deaconess

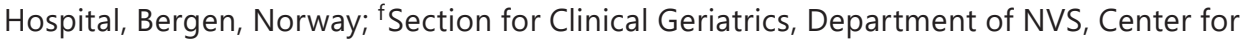
Alzheimer Research, Karolinska Institutet, Stockholm, Sweden
\end{abstract} \\ Hospital, Roskilde, Denmark; ${ }^{b}$ Danish Dementia Research Centre, Department of Neurology,
}

Keywords

Alzheimer disease · Mild cognitive impairment · Biomarker · Quantitative electroencephalography · Diagnosis · Prognosis · Cerebrospinal fluid · Amyloid · Tau protein

\begin{abstract}
Aim: To examine diagnostic and prognostic potential of quantitative electroencephalography (qEEG) analyzed by the statistical pattern recognition (SPR) method in patients with cognitive impairment. We compared the differential diagnostic ability of SPR to visual EEG analysis. Correlation between SPR findings and cerebrospinal fluid (CSF) Alzheimer disease (AD) biomarkers were evaluated. Methods: It is a multicenter cohort study involving 129 patients, (mild cognitive impairment $[\mathrm{MCl}], A D$, and healthy controls). Standardized EEG was performed at baseline. Patients were continuously clinically evaluated. Results: Receiver Operating Characteristic curves showed a low discriminative ability of SPR and no ability to predict clinical progression in patients with $\mathrm{MCl}$. Moderate correlation between SPR analysis and CSF AD biomarkers was found. Conclusion: The diagnostic and prognostic abilities of qEEG were low. The SPR method was superior to the visual EEG analysis. The qEEG method correlates well to CSF $A D$ biomarkers, suggesting association with pathology in $A D$.


Schjønning Nielsen et al.: QEEG Analyzed by SPR as a Diagnostic and Prognostic Tool: Results from a Nordic Multicenter Cohort Study

\section{Introduction}

Cognitive impairment is common among elderly people. The most disabling form of cognitive impairment is dementia. Dementia may be caused by several diseases; however, Alzheimer disease (AD) is the predominant cause $[1,2]$. Although only symptomatic treatment is currently available, it is important to identify those patients affected by cognitive impairment due to $\mathrm{AD}$ and it is equally important to make an early diagnosis to initiate timely treatment, when neuronal damage may still be limited $[3,4]$. This issue becomes even more relevant when potentially disease modifying drugs will be introduced. Mild cognitive impairment (MCI) can be regarded as a risk state for dementia or in some cases as a prodromal stage of a dementia disease, especially AD [5]. It has been reported that $40-60 \%$ of patients with MCI develop AD within 5 years and even $10-15 \%$ of patients with MCI develop AD within 1 year $[6,7]$.

However, not every case of MCI will progress to dementia. MCI patients may remain cognitively stable or even reverse to normal cognition in time [8-10]. Currently, one way to differentiate between patients with MCI due to an underlying neurodegenerative disease and stable MCI patients is to continuously evaluate patients to detect whether they clinically progress to fulfill the criteria for dementia. Efforts are being made to determine early biomarkers of various dementia disorders, which exhibit a high diagnostic accuracy and reflect the biochemical and pathological hallmarks of the disease as well as being able to predict the conversion of MCI to $\mathrm{AD}[3,11]$. Various biomarkers, in particular imaging, for example, Magnetic Resonance Imaging (MRI), Positron Emission Tomography (PET) and Single Photon Emission CT methods, as well as cerebrospinal fluid (CSF) biomarkers have proven helpful in diagnosing $\mathrm{AD}$ and findings from biomarker analyses have even been incorporated in research criteria for $\mathrm{AD}$ [12]. However, these biomarkers may have limited usefulness, as some are expensive and may not be available outside academic centers, or are invasive and therefore may not be widely applied.

Electroencephalography (EEG) is an inexpensive method; it is simple to perform and available in most clinical settings $[13,14]$. Furthermore, the method is noninvasive and thereby implies almost no physical discomfort for the patient. EEG records the electrical activity of cortical neurons and thus reflects the underlying brain function. EEG may thereby potentially be an important biomarker of disturbances in the cognitive function $[15,16]$. As $\mathrm{AD}$ is a disease affecting the cerebral cortex, EEG abnormalities reflecting the structural and functional deficits in cortex due to the disease are frequently shown in AD [17]. Likewise, early EEG changes have been found in subcortical dementias, for example, Dementia with Lewy Bodies (DLB) and Parkinson Disease Dementia (PDD), which may separate DLB and PDD from both AD as well as healthy persons [18]. Currently, there is no clear evidence whether the use of conventional visual EEG analysis is exceeded by quantitative EEG (qEEG) analysis for diagnostic purposes [16].

QEEG is a computer-based analysis, which is independent of the subjective assessment seen in visual rated conventional EEG $[19,20]$. Several studies have demonstrated a high diagnostic value of qEEG when evaluating patients with $\mathrm{AD}$ and $\mathrm{MCI}$ in the general population $[17,21,22]$. As EEG alterations may be present early in the course of the disease and even in the pre-clinical stage, qEEG may indeed be diagnostically and prognostically valuable in patients with MCI [22-26].

Statistical pattern recognition (SPR) is a data-analytic method used in qEEG. The SPR analyses in this study are based on the Icelandic database described in previous publications $[18,27]$, and therefore differs from publications evaluating EEG in dementia and MCI in a more traditional way. 
The method has been found valuable in the diagnostic work-up of patients with cognitive impairment $[18,19,27]$. In particular, SPR discriminated excellently between LBD/PDD and healthy controls (HC) as well as other dementia disorders, but it also exhibited a good discriminative ability between HC and individuals with dementia. However, the diagnostic value of the SRP method in patients with MCI is more uncertain, as conflicting results have been reported $[18,27,28]$. In addition, the prognostic value of the SPR method in a consecutively referred heterogeneous cohort of patients with MCI in a memory clinic setting has not yet been established.

In the present study, the aim was to assess the potential value of the method as a prognostic indicator in a group of patients diagnosed with MCI and to investigate possible associations between the SPR analysis and the CSF biomarker analysis. Furthermore, we aimed to investigate the ability of the SRP method to differentiate between patients with MCI, mild AD and $\mathrm{HC}$ compared to the diagnostic ability of the conventional visual EEG analysis.

\section{Materials and Methods}

\section{Study Population}

The study is a subgroup study of a larger prospective multicenter cohort study, conducted at 5 Nordic memory clinics located at the Zealand University Hospital in Roskilde and University Hospital Copenhagen, Rigshospitalet in Copenhagen (Denmark), the Deaconess Hospital in Bergen and the Oslo University Hospital (Norway), and the Karolinska University Hospital Huddinge in Stockholm (Sweden).

Patients, aged 50-85 years consecutively referred for cognitive evaluation and diagnosed with either MCI or mild AD (baseline Mini-Mental State Examination [MMSE] score of 22 or above) from May 2011 to December 2014 were eligible for inclusion. In this sub-study of the NORDEEG cohort, we included only subjects in whom lumbar puncture with examination of the CSF biomarkers AB42, total tau (T-Tau) and phosphorylated tau (P-tau), had been carried out as part of the standardized clinical diagnostic work-up.

Common standards for the diagnostic work-up were followed in all 5 hospitals. At the time of referral, patients underwent a standardized diagnostic assessment including physical and neurological examination, clinical history from patient and relative, routine blood analysis, brain CT or MRI scan, lumbar puncture with CSF AD biomarker analysis as well as a cognitive screening by a MMSE. If patients remained diagnostically unresolved despite the standardized diagnostic assessment, a full neuropsychological evaluation was conducted by a neuropsychologist.

Patients with MCI were diagnosed according to the Winblad consensus criteria [29]. Patients with AD were diagnosed according to the DSM-IV-R and the NINCDS-ADRDA for AD $[12,30]$.

The general rule for the NORDEEG cohort was that either the diagnoses were settled at a consensus conference by an experienced multidisciplinary team or as a minimum in consensus between at least 2 experienced physicians (specialist level) in the field of dementia, based on all available examination results, but independently ("blind") of the EEG results. Participants were only referred for a standardized EEG, if they had consented to participate in the study.

Patients were excluded if they did not give informed consent to participate or if they were affected by other neurological, physical or severe psychiatric disease. Patients, who also were diagnosed with mild anxiety or depression were included, as these conditions often are seen concurrently in patients with MCI and mild dementia. Patients were also excluded if they were found to use sedative medication (e.g., Morphine, Benzodiazepine on a regular basis), which could give rise to cognitive impairment. 
Box 1.

\begin{tabular}{|ll|}
\hline Classifier index & Definition \\
\hline "Healthy control index" (qEEG HC index) & $\begin{array}{l}\text { Separate healthy individuals from MCI or any } \\
\text { dementia disorder } \\
\text { "MCI" (qEEG MCI index) }\end{array}$ \\
"AD index" (qEEG AD index) & $\begin{array}{l}\text { Separate MCI from any dementia disorder } \\
\text { disorder } \\
\text { "AD, moderate/severe index" (qEEG ADms index) }\end{array}$ \\
$\begin{array}{l}\text { Separate moderate to severe AD from MCI or } \\
\text { any other dementia disorder } \\
\text { "DLB/PDD index" (qEEG DLB/PDD index) }\end{array}$ & $\begin{array}{l}\text { Separate DLB and PDD from MCI or any other } \\
\text { dementia disorder }\end{array}$ \\
\hline MCI, mild cognitive impairment; AD, Alzheimer's disease; DLB, Dementia with Lewy Bodies; PDD, \\
Parkinson Disease Dementia.
\end{tabular}

Patients were clinically evaluated yearly on an ongoing basis as a part of the normal clinical routine in the memory clinics and diagnoses were reevaluated and based on clinical progression, the diagnoses were subsequently revised by experienced physicians.

As the study is a naturalistic study, in which patients are recruited and clinically followed as a part of everyday clinical practice, the length of the follow-up period varied due to differences in the clinical set-ups, due to differences in patients' ongoing ability to perform cognitive testing as well as unexpected dropouts.

The HC were volunteers aged 50-85 years recruited for scientific research through public invitations at the memory clinics, at local associations for elderly and through an online recruitment site for trail subjects. Exclusion criteria were presence of memory complaints or other cognitive symptoms as well as signs of major neurological, psychiatric, or physical disease, which potentially could elicit cognitive impairments. Furthermore, they could not receive sedatives (Morphine and Benzodiazepine) on a regular basis. The $\mathrm{HC}$ all underwent the previously described standardized diagnostic assessment as the patients, including EEG, in either the memory clinic at Zealand University Hospital or at University Hospital Copenhagen, Rigshospitalet (Denmark) at inclusion. Follow-up visits were carried out on a yearly basis for 3 years, where all HC had serial cognitive screening.

The investigator, who performed the follow-up visits, was blinded to the results of EEG and CSF analysis.

\section{Electroencephalogram}

EEG registrations were performed at all 5 clinics according to a common harmonized instruction. The EEG recording and feature extraction are comprehensively described by Engedal et al. [27]. EEG data were analyzed by the SPR technique based on a dementia database developed by MentisCura [31].

In essence, the SPR technique compares EEG recordings with normative data from a database containing EEG recordings from more than 1,000 patients with dementia disorders as well as approximately $500 \mathrm{HC}$. By comparing features from 2 diagnostic groups of EEGs, a classifier is constructed. When an individual EEG recording is analyzed according to the classifiers, it yields an index for each classifier with a value between 0 and 1 . The index values represent the likeliness of the individual EEG to be indistinguishable from either one of the diagnostic groups. If the value is close to 0 subsequently to 1 , the EEG is indistinguishable from EEGs in one of the diagnostic groups in question $[18,28]$. Five classifiers from a previous study were used in the present study (Box 1; Fig. 1) [18]. Subsequently, all EEGs were evaluated at 
Schjønning Nielsen et al.: QEEG Analyzed by SPR as a Diagnostic and Prognostic Tool: Results from a Nordic Multicenter Cohort Study

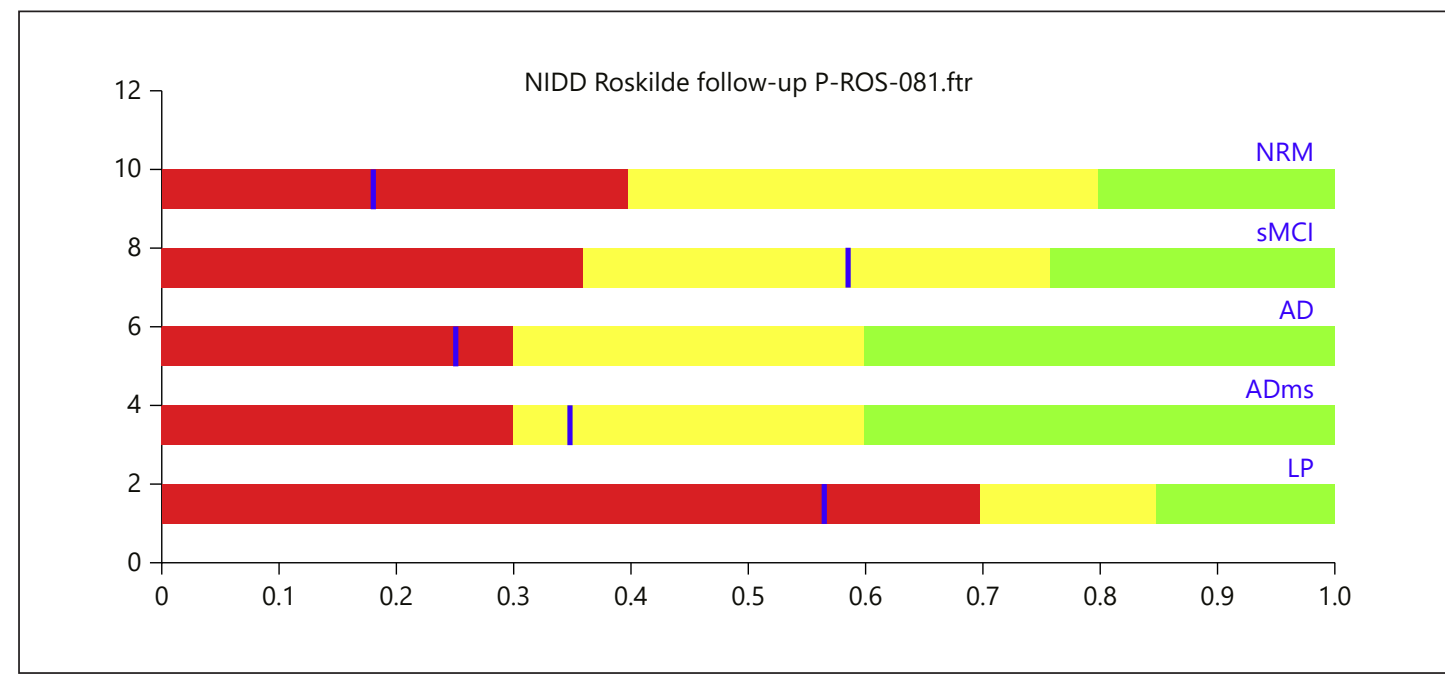

Fig. 1. An example of the SPR-EEG analysis according to the 5 classifiers. The blue line represents the likeliness of the EEG to be indistinguishable from either one of the diagnostic groups constituting the indices. The indices are explained in Box 1. NRM, Healthy control index, SMCI, Mild Cognitive impairment index, AD, Alzheimer's disease index, ADms, Alzheimer's disease, moderate/severe index, LP, Dementia Lewy Body/ Parkinson Disease Dementia index.

each participating clinic by one certified medical doctor and classified on the basis of the index values of each classifier produced by the SPR technique. This evaluation was performed without knowledge of the clinical diagnosis. A final EEG based diagnosis was established if there was consensus among the certified medical doctors at each participating clinics with regard to the classification of the individual EEG among the participating clinics. In a fraction of the qEEGs, it was not possible to classify the pattern according to one of the 4 previously defined classifiers. These were combined in a group with an "inconclusive" qEEG pattern.

To evaluate the diagnostic and prognostic value of the SPR method regardless of the specific qEEG diagnosis, we used the individual qEEG diagnoses to separate the qEEGs into 2 categories. A stable category, which embraced patients with non-progressive qEEG patterns classified as "Normal," and "MCI" and a progressive category embracing patients with progressive qEEG patterns classified as "AD" and "DLB/PDD."

\section{Visual EEG Assessment}

In addition to the SPR analysis, visual EEG assessments were also performed to register focal abnormalities as well as paroxysmal alterations. The EEG recordings were assessed by 1 of 2 experienced neurophysiologists (MDA and TWK). At the time of the assessment, they did not have any knowledge of diagnosis and other clinical information.

\section{CSF Analysis}

Lumbar puncture was performed at all 5 clinics. Methods for collection and analysis are well described by Ferreira et al. [32]. Levels of CSF Amyloid (A $\beta_{42)}$, T-tau and P-tau were analyzed and regarded as markers of amyloid accumulation subsequent neurodegeneration.

\section{Ethics}

The study was reported and approved by the Data Protection Agency Authorities and by the Regional Ethical Committees in each of the participating countries. All participants signed an informed consent form prior to participation. 
Table 1. Characteristics of the study population baseline

\begin{tabular}{lccc}
\hline Total study population & HC & MCI & AD \\
\hline$n(\%)$ & $41(31.8)$ & $56(43.4)$ & $32(24.8)$ \\
Age, years & $65.0(62.0-71.5)$ & $68.0(64.0-74.0)$ & $69.5(64.5-75.6)$ \\
$\quad$ Age, range & $50-85$ & $58-82$ & $58-85$ \\
Gender (M:F), \% & $53.7: 46.3$ & $53.6: 46.4$ & $68.75: 31.25$ \\
Education, years & $13.0(10.0-16.0)$ & $12.0(9.0-15.0)$ & $10.5(8.0-14.8)$ \\
Follow-up, months & $35.0(34.5-36.0)$ & $22.0(15.0-32.0)$ & $15.0(11.0-27.0)$ \\
MMSE score* & $29(28-30)$ & $28(26-29)$ & $25(24-27)$ \\
Comorbidity, \%* & $4.9(25.0$ & 18.8 \\
CSF Amyloid & $916.0(805.0-1166.0)$ & $715.0(563.0-1037.0)$ & $574.5(374.0-668.0)^{* *}$ \\
CSF P-tau & $47.0(35.0-64.0)$ & $58.0(39-74.0)$ & $84.0(70.5-94.8)^{* *}$ \\
CSF T-tau & $275.0(191.0-346.0)$ & $394.0(223.5-542.5)$ & $556.5(470.0-893.8)^{* *}$ \\
\hline
\end{tabular}

Data are expressed as medians (IQRs), significant when $p<0.01$, due to multiple comparisons. Comorbidity covers depression or anxiety.

* Significant, when all groups are compared individually.

** Significant, when compared to HC and MCI.

MMSE, mini-mental state examination; CSF, cerebrospinal fluid; P-tau, phosphorylated tau protein; T-tau, total tau protein; MCI, mild cognitive impairment, AD, Alzheimer disease.

\section{Statistics}

Kruskall-Wallis test and Mann-Whitney U test were used for median comparisons between groups subsequent for pairwise comparisons. The chi-square test was used for nominal variables. To correct for analysis with multiple comparisons, results were considered statistically significant when $p$ values were $p<0.01$. Receiver Operating Characteristic (ROC) curves of the qEEG diagnosis, qEEG categories and the visual EEG analysis were drawn as a plot of the true positive value (sensitivity) against the false positive value (1 - specificity) for different possible cutoff values of the respective markers. The ROC curves illustrate the ability of each parameter to (1) separate both the HC from the cognitive impaired patients as well as separate MCI from AD and (2) separate the progressive versus the stable MCI patients. The area under the curve (AUC) was calculated as a measure of the parameter's overall accuracy. Gamma correlations were used to study relations between variables.

Statistical analysis was carried out using SPSS version 22 and "R" environment for statistical computing, version 3.1.2.

\section{Results}

A total of 129 participants were included in our study. At baseline, 56 were diagnosed with MCI, 32 were diagnosed with mild AD, and 41 were enrolled as HC. Clinical data are presented in Table 1.

Apart from expected differences in cognitive function and biomarker status, a significant difference in comorbidity between the groups was found, as $25 \%$ in the MCI group were affected by depression or anxiety compared to $18.8 \%$ in the AD group and only $4.9 \%$ in the HC group. A total of $90 \%$ of the patients with a final consensus diagnosis of AD had 1 or more abnormal CSF biomarkers, while $65 \%$ of the clinical MCI group and $24 \%$ of the HC group had one or more abnormal CSF biomarkers.

The qEEG diagnoses and the qEEG categories distributed by clinical diagnosis are shown in Table 2. In the HC group, a remarkable low percentage of approximately $15 \%$ had a qEEG 
Table 2. qEEG characteristics in the study population

\begin{tabular}{|c|c|}
\hline \multicolumn{2}{|c|}{ Dement Geriatr Cogn Disord Extra 2018;8:426-438 } \\
\hline DOI: $10.1159 / 000490788$ & $\begin{array}{l}\text { (c) } 2018 \text { The Author(s). Published by S. Karger AG, Basel } \\
\text { www.karger.com/dee }\end{array}$ \\
\hline
\end{tabular}

Schjønning Nielsen et al.: QEEG Analyzed by SPR as a Diagnostic and Prognostic Tool: Results from a Nordic Multicenter Cohort Study

\begin{tabular}{lccc}
\hline & HC & MCI & AD \\
\hline $\begin{array}{l}\text { Total study population, } n \\
\text { qEEG diagnosis, } n \text { (\%) }\end{array}$ & 41 & 56 & 32 \\
$\quad$ Normal & $6(14.6)$ & $9(16.1)$ & $1(3.1)$ \\
MCI & $11(26.8)$ & $12(21.4)$ & $2(6.3)$ \\
AD & $13(31.7)$ & $22(39.3)$ & $22(68.8)$ \\
LBD/PPD & $1(2.4)$ & $3(5.4)$ & $1(3.1)$ \\
Inconclusive & $10(24.4)$ & $10(17.9)$ & $6(18.8)$ \\
qEEG categories, $n(\%)^{*}$ & & & \\
$\quad$ Stable & $17(41.5)$ & $21(37.5)$ & $3(9.4)^{*}$ \\
$\quad$ Progressive & $14(34.1)$ & $25(44.6)$ & $23(71.9)^{*}$ \\
$\quad$ Inconclusive & $10(24.4)$ & $10(17.9)$ & $6(18.8)$ \\
\hline
\end{tabular}

Stable qEEG pattern is comprised by qEEGs with normal, SCI and MCI patterns. Progressive qEEG pattern is comprised by qEEGs with $\mathrm{AD}$ and LBD patterns.

* Significant, when compared to MCI and HC, $p<0.05$.

$\mathrm{MCI}$, mild cognitive impairment; AD, Alzheimer's disease; $\mathrm{HC}$, healthy control; LBD, Lewy Body Dementia; PDD, Parkinson Disease Dementia.

Table 3. Characteristics of the MCI group

\begin{tabular}{lcc}
\hline Population characteristics & Clinical stable MCI & Clinical progressive MCI \\
\hline$n(\%)$ & $21(47.7)$ & $23(52.3)$ \\
MMSE score & $28(26-29)$ & $27(25-29)$ \\
CSF Amyloid & $699.0(549.0-998.5)$ & $660.0(468.5-913.5)$ \\
CSF T-tau & $414.0(244.0-607.0)$ & $441.0(327.0-601.5)$ \\
CSF P-tau & $61.0(39.0-88.5)$ & $58.0(45.5-74.0)$ \\
Stable qEEG pattern, $n(\%)$ & $6(28.6)$ & $6(26.1)$ \\
Progressive qEEG pattern, $n(\%)$ & $10(47.6)$ & $13(56.5)$ \\
Inconclusive qEEG pattern, $n(\%)$ & $5(23.8)$ & $4(17.4)$ \\
\hline
\end{tabular}

Progressive qEEG pattern is comprised of qEEGs with AD and LBD patterns. Stable qEEG pattern is comprised of qEEGs with normal and MCI patterns.

diagnosis of "normal" while 31.7\% had a SPR-EEG diagnosis of "AD" and 24.4\% were "inconclusive." Significantly more patients diagnosed with AD had a progressive qEEG pattern at baseline than MCI patients and $\mathrm{HC}$ respectively (Table 3). On the contrary, only $9.4 \%$ in the AD group had a stable qEEG pattern compared to 37.5 and $41.5 \%$ in the MCI group and HC respectively. The diagnostic accuracy of the SPR method to discriminate between MCI and HC respectively AD as well as between AD and HC was illustrated by ROC curve analysis (Table 4).

A subgroup of 84 participants had data on the visual analysis of the conventional EEG available (Table 2). Interestingly, by visual inspection, $28 \%$ of the $\mathrm{MCI}$ patients exhibited focal abnormalities compared to only 5 and $2.6 \%$ in the AD patients and $\mathrm{HC}$ respectively $(p<0.01$, Cramer's $V=0.375)$. No participants exhibited paroxysmal abnormalities at their baseline EEG recording (Table 2). ROC curve analyses were also constructed to illustrate the diagnostic accuracy of the visual EEG analyses compared to the SPR method in the subgroup (Table 4). 
Table 4. ROC curves analysis of the qEEG diagnosis, qEEG categories, and the visual EEG analysis

\begin{tabular}{llll}
\hline Group comparisons & $\begin{array}{l}\text { AUC } \\
\text { qEEG diagnosis }\end{array}$ & $\begin{array}{l}\text { AUC } \\
\text { qEEG categories }\end{array}$ & $\begin{array}{l}\text { AUC } \\
\text { Visual analysis }\end{array}$ \\
\hline Total study group & & & \\
$\quad$ MCI versus HC & 0.56 & 0.56 \\
AD versus MCI & 0.60 & 0.61 & \\
AD versus HC & 0.66 & 0.66 & \\
Subgroup with visual EEG analysis & & & 0.63 \\
MCI versus HC & 0.67 & 0.64 & 0.36 \\
AD versus MCI & 0.54 & 0.58 & 0.49 \\
AD versus HC & 0.69 & 0.70 & \\
\hline
\end{tabular}

ROC, receiver operating characteristic; MCI, mild cognitive impairment; AD, Alzheimer's Disease; HC, healthy controls; AUC, area under the curve.

Prognostic Value of the SPR Method in MCI

During the follow-up period, 23 (41.1\%) participants with MCI clinically progressed to fulfill the diagnostic criteria for dementia. In this group of progressive MCI patients, 1 participant was diagnosed with Lewy Body dementia, 1 participant was diagnosed with corticobasal degeneration, and the remaining 21 participants were diagnosed with AD. Twenty-one participants (37.5\%) with MCI remained stable and the remaining 12 participants $(21.4 \%)$ were lost to follow-up. Clinical data are presented in Table 3.

No significant difference in cognitive status, that is, MMSE score, or in CSF AD biomarkers between the stable and progressive groups was seen. At baseline no significant differences in qEEG categories between the clinical stable and progressive MCI groups was found.

ROC curves analysis revealed no predictive ability with regard to predicting future clinical progression in our group of MCI patients with an AUC value for qEEG diagnosis of 0.58 respectively an AUC value for qEEG categories of 0.55 .

Correlations between the SPR Method and Cognitive Tests as Well as CSF AD Biomarkers

In the entire cohort, a moderate negative correlation between the SRP-EEG diagnosis as well as the qEEG categories and both the baseline MMSE score and CSF Amyloid was found. In addition, a moderate positive correlation between the qEEG diagnosis as well as the qEEG categories and CSF T-tau respectively CSF P-tau levels were found (Table 4).

\section{Discussion}

Our findings with regard to a low diagnostic and prognostic ability of the SPR method in a typical clinical setting were unexpected, given the fact that previous studies showed more promising results $[18,27,32,33]$.

The diagnostic and prognostic use of qEEG in the diagnostic assessment of patients with cognitive impairment has gained much attention. In particular, the ability of qEEG to diagnose dementia disorders in the very early phase of the disease, even in the pre-dementia phase, where the patients only fulfill the criteria of MCI, has been an area of interest.

Previous studies have demonstrated an excellent to good discriminative ability of the SPR based on the Islandic dementia database in separating AD from HC $[18,27,28]$. Nevertheless, conflicting results have been reported as Ommundsen et al. [19] found the method to exhibit a somewhat lower ability to diagnose AD in patients referred to a memory clinic. 
Likewise, Snaedal et al. [18] found the SPR method to separate AD from stable MCI with an accuracy of $80 \%$ indicating that the stable MCI patients exhibit an EEG pattern quite different from AD. However, this could not be replicated in a more recent study by Engedal et al. [27].

The low differential diagnostic ability of the method in our study was unexpected regarding these more favorable results. We find no critical differences in the methodology used in the present study and in previous studies to explain this, although the SPR classifiers may have been slightly different. For a full technical description of the applied SPR method and the selection of qEEG classifiers, please see reference [18].

However, it may be contributed to the low specificity of the SPR to identify the healthy subjects in our study population. The method did only classify $14.6 \%$ of the $\mathrm{HC}$ as having a "normal" qEEG pattern, whereas $34.1 \%$ were classified as having a progressive qEEG pattern, that is, an AD or DLB/PDD qEEG pattern and $24.4 \%$ had an inconclusive qEEG pattern (Table 2).

Therefore, one may wonder whether our group of HC was not comparable with the HC group used to create the database. However, there were no differences in the age range between the 2 groups. Likewise, we find no evidence of our HC group being less healthy than the $\mathrm{HC}$ group in the database, as our $\mathrm{HC}$ group underwent a rather comprehensive diagnostic assessment including multiple cognitive testing and CT or MRI scan as well as a lumbar puncture with CSF AD biomarker analysis and an amyloid PET scan in the majority of the subjects. During the baseline assessment, we found only 2 healthy subjects, who had signs of cerebral amyloid (one had low CSF amyloid level and one had a positive amyloid PET scan but a normal CSF amyloid level). However, both were without any cognitive complaints and performed in the normal range on all cognitive tests. The influence of differences in mental comorbidity cannot be ruled out, as it may affect the EEG patterns, causing an inconclusive EEG pattern. Otherwise, the low discriminative ability found in our study population cannot be explained.

Nevertheless, the SPR method exhibited a moderate to good sensitivity as $71.9 \%$ of all AD patients were classified correctly as having a progressive qEEG pattern.

In accordance with the study by Engedal et al. [27] and Ommundsen et al. [19], we found the SPR method to separate patients with AD from MCI to be not so effective, thereby indicating that a proportion of the MCI patients may actually be affected by AD in the early stage. The somewhat higher discriminative ability in separating AD from MCI found by Snaedal et al. [18] may partly be due by the fact that they only included MCI patients, who prior to inclusion had remained clinical stable for a minimum of 2 years.

As the SPR method failed to discriminate between $\mathrm{HC}$ and patients with both MCI and AD in our population, we hypothesized that a standardized visual analysis of a conventional EEG may potentially have a similar or superior diagnostic accuracy. It is well known that signs of diffuse slow EEG rhythms is useful in separating HC from AD [34, 35], but the value of focal EEG alterations in cognitively impaired is not clear [36]. Liedorp et al. [36] found that focal abnormalities without diffuse alterations were associated with $\mathrm{MCI}$, whereas the combination of focal and diffuse alterations argued against the MCI diagnosis. This is in line with the finding by Visser et al. [37] that focal EEG changes in healthy persons may be an early sign of cognitive dysfunction.

Our findings were supportive of the previous studies, as we found a significant higher percentage of focal EEG abnormalities in the MCI group than in the group of AD patients and HC (Table 2). Nevertheless, ROC analysis revealed that the visual assessment of EEG performed poorly in discriminating the groups, and worse than the computerized analysis (qEEG).

Specific EEG alterations may potentially be predictive of future clinical progression in MCI patients to fulfill the diagnostic criteria of $\operatorname{AD}[17,22,24,38]$. These EEG alterations may resemble the EEG abnormalities typically found in $\mathrm{AD}$, for example, an increase in theta and 
Table 5. Correlations (gamma) between the SPR method and CSF AD biomarkers in the entire cohort
Schjønning Nielsen et al.: QEEG Analyzed by SPR as a Diagnostic and Prognostic Tool: Results from a Nordic Multicenter Cohort Study

\begin{tabular}{lll}
\hline Parameters & qEEG diagnosis & qEEG categories \\
\hline CSF amyloid & $r=-0.254, p<0.01$ & $r=-0.265, p<0.01$ \\
CSF T-tau & $r=0.163, p<0.05$ & $r=0.167, p=0.05$ \\
CSF P-tau & $r=0.187, p<0.05$ & $r=0.209, p<0.05$ \\
MMSE score & $r=-0.210, p<0.05$ & $r=-0.230, p<0.05$ \\
\hline
\end{tabular}

The qEEG diagnosis is defined as the individually qEEG diagnosis based on the result of the classifier analysis. The qEEG categories is based on the qEEG diagnosis and each group represent patients with qEEGs exhibiting either a stable qEEG pattern, a progressive qEEG pattern, or an inconclusive pattern.

MMSE, mini-mental state examination; CSF, cerebrospinal fluid; P-tau; phosphorylated tau protein; T-tau, total tau protein.

delta activities as well as a decrease in alpha and beta activities [17]. In a pilot study, Snaedal et al. [28] demonstrated a potential ability of the SPR method in predicting clinical progression to AD in a group of MCI patients Likewise, a recent Italian study of MCI patients showed that qEEG may predict future clinical development of LBD [26]. In contrast, others have not detected EEG alterations, which could differentiate between the progressive and the stable MCI patients [24, 25].

In our study, the distributions of patients with stable and progressive qEEG patterns were very similar in the clinical stable and progressive group of MCI patients (Table 3). Hence, ROC curve analyses demonstrated a low predictive ability of future clinical progression in MCI in our study.

This finding is in contrast with previous findings by Snaedal et al. [18]. The conflicting results may partly be explained by differences in study populations as Snaedal et al. [18] exclusively compared AD with MCI patients remaining stable for 2 years or more, while we embraced all patients diagnosed with $\mathrm{MCI}$ at baseline. As the rate of cognitive decline and clinical progression in dementia disorders varies, this might in our study imply inclusion of patients in the stable MCI group, who in fact are affected by AD in the prodromal stage and hence in time will progress to a dementia disorder. This could explain the high percentage of stable MCI patients exhibiting a progressive qEEG pattern at baseline. Supporting this is the finding that there are no significant group differences in the CSF AD biomarker levels at baseline between the progressive and stable $\mathrm{MCI}$ patients (Tables 3,5), as correlations between CSF AD biomarkers and EEG alterations have been demonstrated both within our study as well as in others [39].

\section{Strengths}

Our study has several strengths. First, it was conducted in a memory clinic setting using a consecutively recruited population. All patients were examined in a standardized manner according to harmonized protocol used by all participating clinics. The patients were followed prospectively, which eliminated biases from retrospective information gathering. Furthermore, all EEG recordings were visually analyzed by 1 of 2 experienced neurophysiologists, which minimized interrater variability. Finally, the EEG recordings were not part of the diagnostic assessment, thereby reducing the risk of circularity.

\section{Limitations}

The fairly small sample size of this study is a limitation. The findings of low predictive ability of the SPR method with regard to progression may as a consequence be due to lack of 
statistical power. Likewise, the variability in the length of the follow-up period was large, which may have influenced the predictive value of the SPR method. In particular, this is an issue with regard to the group of stable MCI patients as it is well known that time to conversion varies and it is therefore likely that some patients labeled stable at the end of our study, will eventually progress. Finally, we cannot rule out that some patients may have been diagnosed incorrectly, as the cohort lacks postmortem diagnostic confirmation. To address this, the clinical diagnosis was established by at least 2 experienced physicians or by a multidisciplinary team at each clinic.

\section{Conclusion}

In conclusion, we found the overall diagnostic ability of the SPR method to be low in our consecutively referred cohort. The method exhibited the highest discriminative ability in separating AD from HC. Nevertheless, it was still lower than reported in other studies. We did find the SPR method to be superior to the conventional visual EEG analysis in discriminating between both the $\mathrm{HC}$ and all groups of the cognitively impaired patients. The low ability of the SPR method to predict future clinical progression in patients with MCI in our cohort was unexpected as previous studies have indicated more promising results. Further prospective studies on the utility of the SPR method for predicting clinical progression in a consecutively referred cohort in a normal clinical setting are needed.

\section{Acknowledgments}

The work was supported by grants from the Velux Foundations.

\section{Disclosure Statement}

The authors declare no conflicts of interest.

The SPR technique was developed by MentisCura. However, MentisCura had no influence on the process of data analysis or the conclusions in this paper.

\section{References}

1 World Health Organization. Dementia: a public health priority. Dementia. 2012; 112.

2 Danish Health and Medicin Authority. National Klinisk Retningslinje om udredning og behandling af demens. 2013.

3 Sperling RA, Jack CR Jr, Aisen PS. Testing the right target and right drug at the right stage. Sci Transl Med. 2011 Nov;3(111):111cm33.

4 Waldemar G, Phung KT, Burns A, Georges J, Hansen FR, Iliffe S, et al. Access to diagnostic evaluation and treatment for dementia in Europe. Int J Geriatr Psychiatry. 2007 Jan;22(1):47-54.

5 Gauthier S, Reisberg B, Zaudig M, Petersen RC, Ritchie K, Broich K, et al.; International Psychogeriatric Association Expert Conference on mild cognitive impairment. Mild cognitive impairment. Lancet. 2006 Apr; 367(9518):1262-70.

6 Hampel H, Teipel SJ, Fuchsberger T, Andreasen N, Wiltfang J, Otto M, et al. Value of CSF beta-amyloid1-42 and tau as predictors of Alzheimer's disease in patients with mild cognitive impairment. Mol Psychiatry. 2004 Jul; 9(7): 705-10.

7 Hansson O, Zetterberg H, Buchhave P, Londos E, Blennow K, Minthon L. Association between CSF biomarkers and incipient Alzheimer's disease in patients with mild cognitive impairment: a follow-up study. Lancet Neurol. 2006 Mar;5(3):228-34.

8 Ganguli M, Dodge HH, Shen C, DeKosky ST. Mild cognitive impairment, amnestic type: an epidemiologic study. Neurology. 2004 Jul;63(1):115-21. 
9 Palmer K, Wang HX, Bäckman L, Winblad B, Fratiglioni L. Differential evolution of cognitive impairment in nondemented older persons: results from the Kungsholmen Project. Am J Psychiatry. 2002 Mar;159(3):43642.

10 Solfrizzi V, Panza F, Colacicco AM, D’Introno A, Capurso C, Torres F, et al.; Italian Longitudinal Study on Aging Working Group. Vascular risk factors, incidence of MCI, and rates of progression to dementia. Neurology. 2004 Nov;63(10):1882-91.

11 Solfrizzi V, D'Introno A, Colacicco AM, Capurso C, Todarello O, Pellicani V, et al. Circulating biomarkers of cognitive decline and dementia. Clin Chim Acta. 2006 Feb;364(1-2):91-112.

12 Dubois B, Feldman HH, Jacova C, Dekosky ST, Barberger-Gateau P, Cummings J, et al. Research criteria for the diagnosis of Alzheimer's disease: revising the NINCDS-ADRDA criteria. Lancet Neurol. 2007 Aug;6(8):734-46.

13 Kowalski JW, Gawel M, Pfeffer A, Barcikowska M. The diagnostic value of EEG in Alzheimer disease: correlation with the severity of mental impairment. J Clin Neurophysiol. 2001 Nov;18(6):570-5.

14 Engedal K, Oftedal SI, Lilleaas F, Laake P. Electroencephalography, visual evoked potentials, and cerebral CAT-scan as diagnostic tools in senile dementia of Alzheimer type. Aging (Milano). 1989;1(2):139-45.

15 Jelic V, Kowalski J. Evidence-based evaluation of diagnostic accuracy of resting EEG in dementia and mild cognitive impairment. Clin EEG Neurosci. 2009 Apr;40(2):129-42.

16 Nuwer M. Assessment of digital EEG, quantitative EEG, and EEG brain mapping: report of the American Academy of Neurology and the American Clinical Neurophysiology Society. Neurology. 1997 Jul;49(1):27792.

17 Jeong J. EEG dynamics in patients with Alzheimer's disease. Clin Neurophysiol. 2004 Jul;115(7):1490-505.

18 Snaedal J, Johannesson GH, Gudmundsson TE, Blin NP, Emilsdottir AL, Einarsson B, et al. Diagnostic accuracy of statistical pattern recognition of electroencephalogram registration in evaluation of cognitive impairment and dementia. Dement Geriatr Cogn Disord. 2012;34(1):51-60.

19 Ommundsen N, Engedal K, Øksengård AR. Validity of the quantitative EEG statistical pattern recognition method in diagnosing Alzheimer's disease. Dement Geriatr Cogn Disord. 2011;31(3):195-201.

20 Brenner RP, Reynolds CF 3rd, Ulrich RF. Diagnostic efficacy of computerized spectral versus visual EEG analysis in elderly normal, demented and depressed subjects. Electroencephalogr Clin Neurophysiol. 1988 Feb;69(2):110-7.

21 Babiloni C, Ferri R, Binetti G, Vecchio F, Frisoni GB, Lanuzza B, et al. Directionality of EEG synchronization in Alzheimer's disease subjects. Neurobiol Aging. 2009 Jan;30(1):93-102.

22 Huang C, Wahlund L, Dierks T, Julin P, Winblad B, Jelic V. Discrimination of Alzheimer's disease and mild cognitive impairment by equivalent EEG sources: a cross-sectional and longitudinal study. Clin Neurophysiol. 2000 Nov;111(11):1961-7.

23 Luckhaus C, Grass-Kapanke B, Blaeser I, Ihl R, Supprian T, Winterer G, et al. Quantitative EEG in progressing vs stable mild cognitive impairment (MCI): results of a 1-year follow-up study. Int J Geriatr Psychiatry. 2008 Nov;23(11):1148-55.

24 Jelic V, Johansson SE, Almkvist O, Shigeta M, Julin P, Nordberg A, et al. Quantitative electroencephalography in mild cognitive impairment: longitudinal changes and possible prediction of Alzheimer's disease. Neurobiol Aging. 2000 Jul-Aug;21(4):533-40.

25 Rossini PM, Del Percio C, Pasqualetti P, Cassetta E, Binetti G, Dal Forno G, et al. Conversion from mild cognitive impairment to Alzheimer's disease is predicted by sources and coherence of brain electroencephalography rhythms. Neuroscience. 2006 Dec;143(3):793-803.

26 Bonanni L, Perfetti B, Bifolchetti S, Taylor JP, Franciotti R, Parnetti L, et al. Quantitative electroencephalogram utility in predicting conversion of mild cognitive impairment to dementia with Lewy bodies. Neurobiol Aging. 2015 Jan;36(1):434-45.

27 Engedal K, Snaedal J, Hoegh P, Jelic V, Bo Andersen B, Naik M, et al. Quantitative EEG Applying the Statistical Recognition Pattern Method: A Useful Tool in Dementia Diagnostic Workup. Dement Geriatr Cogn Disord. 2015;40(1-2):1-12.

28 Snaedal J, Johannesson GH, Gudmundsson TE, Gudmundsson S, Pajdak TH, Johnsen K. The use of EEG in Alzheimer's disease, with and without scopolamine - a pilot study. Clin Neurophysiol. 2010 Jun;121(6):83641.

29 Winblad B, Palmer K, Kivipelto M, Jelic V, Fratiglioni L, Wahlund LO, et al. Mild cognitive impairment-beyond controversies, towards a consensus: report of the International Working Group on Mild Cognitive Impairment. J Intern Med. 2004 Sep;256(3):240-6.

30 McKhann GM, Knopman DS, Chertkow H, Hyman BT, Jack CR Jr, Kawas CH, et al. The diagnosis of dementia due to Alzheimer's disease: recommendations from the National Institute on Aging-Alzheimer's Association workgroups on diagnostic guidelines for Alzheimer's disease. Alzheimers Dement. 2011 May;7(3):263-9.

31 Gudmundsson S, Runarsson TP, Sigurdsson S, Eiriksdottir G, Johnsen K. Reliability of quantitative EEG features. Clin Neurophysiol. 2007 Oct;118(10):2162-71.

32 Ferreira D, Jelic V, Cavallin L, Oeksengaard AR, Snaedal J, Høgh P, et al. Electroencephalography Is a Good Complement to Currently Established Dementia Biomarkers. Dement Geriatr Cogn Disord. 2016;42(1-2): 80-92.

33 Johannesson GH, Snaedal J, Blin NP, Emilsdottir AL, Helgadottir H, Gudmundsson TE, et al. Clinical applicability of EEG in differential diagnosis of dementia. Alzheimers Dement. 2010;6(4):S10. 
34 Claus JJ, Strijers RL, Jonkman EJ, Ongerboer de Visser BW, Jonker C, Walstra GJ, et al. The diagnostic value of electroencephalography in mild senile Alzheimer's disease. Clin Neurophysiol. 1999 May;110(5):825-32.

35 Strijers RL, Scheltens P, Jonkman EJ, de Rijke W, Hooijer C, Jonker C. Diagnosing Alzheimer's disease in community-dwelling elderly: a comparison of EEG and MRI. Dement Geriatr Cogn Disord. 1997 May-Jun;8(3): 198-202.

36 Liedorp M, van der Flier WM, Hoogervorst EL, Scheltens P, Stam CJ. Associations between patterns of EEG abnormalities and diagnosis in a large memory clinic cohort. Dement Geriatr Cogn Disord. 2009;27(1):18-23.

37 Visser SL, Hooijer C, Jonker C, Van Tilburg W, De Rijke W. Anterior temporal focal abnormalities in EEG in normal aged subjects; correlations with psychopathological and CT brain scan findings. Electroencephalogr Clin Neurophysiol. 1987 Jan;66(1):1-7.

38 Prichep LS. Quantitative EEG and electromagnetic brain imaging in aging and in the evolution of dementia. In Annals of the New York Academy of Sciences; 2007. pp. 156-67.

39 Kramberger MG, Kåreholt I, Andersson T, Winblad B, Eriksdotter M, Jelic V. Association between EEG abnormalities and CSF biomarkers in a memory clinic cohort. Dement Geriatr Cogn Disord. 2013;36(5-6):319-28. 